\title{
III. Geburtshülflich-gynäkologische Mitteilungen aus anderen
} Gesellschaften und Vereinen

I. Medizinischer Verein in Greifswald.

(Dtsch. med. Woch. 46. S. 2154). $\delta$. Mai. Kramer spricht über die Indikationen zu den endovesikalen Operationen und ihre natürlíchen Grenzen beím Weibe.

Buchanzeige.

A. Grossich. Meine Pråparationsmethode des Operationsfeldes mittels Jod-tiriktur. Wien 1911. Urban und Schwarzenberg.

Verf. hat zuerst 1907 bei akzidentellen Wunden, dann bei alien über-haupt sich bietenden Operationen die Haut mit Jodtinktur präpariert. Nachdem er dann erprobt hat, daß für einen wirklich idealen Verlauf der Wundheilung das Nichtwaschen vor der Anwendung des Jod geradezu Bedingung ist, verwendet er bei dem großen Material des Bürgerspitals in Fiume diese Methode ausschließlich. Gr. hat die Genugtuung, daß an seine Mitteilung im Zentralbl. f. Chir. 1908 sich eine weitgehende kritische Prüfung des Verfahrens anschloß. Jetzt darf er aus der Summe der ent-sprechenden Publikationen den sicheren Schluß ziehen, daß sein Yerfahren für viele das Verfahren der Wahl geworden ist.

Die klinischen Erfahrungen haben gezeigt, daß die Jodtinktur, auf die ungewaschene, trocken abrasierte Haut gestrichen, dieselbe in einen solchen Zustand versetzt, daß von ihr eine Infektion der Wunde absolut ausgeschlossen ist, also die Haut sterilisiert ist. Eine wichtige Vorbedingung ist, daß die Haut trocken sei. Nur wenn ein nasser Schmutz die Haut bedeckt, muß die Haut vorher mit Alkohol gereinigt werden. In dringlichen Fallen ist diese Grossichsche Methode unersetzlich. Bei aseptischen Fallen gilt sie vielen als die beste. Wundstörungen sind immer auf Infektionen zurückzuführen, deren Ursachen in der Regel leicht und sicher festzustellen sind. Jedenfalls bezeichnet auch B-ef. aus eigner Erfahrung das Verfahren als einen wirklichen Fortschritt auf dem Gebiete der Operationsfeld-Vorbereitung. A. Martin. 